\title{
Propuesta de Valoración del Comportamiento Como Complemento a la Evaluación Emocional de los Usuarios Mientras Interactúan con Sitios Web
}

\section{Behavioral Assessment Proposal to Supplement the Emotional EVALUATION OF UseRS AS THEY INTERACT WITH WeBSITES}

\section{Yenny Méndez Alegría', César A. Collazos² ${ }^{2}$ Toni Granollers³ ${ }^{3}$ Rosa Gil ${ }^{4}$}

Escuela de Ciencias Básicas, Tecnología e Ingeniería, Universidad Nacional Abierta y a Distancia, Cali, Colombia yenny.mendez@unad.edu.co

2Departamento de Sistemas, Universidad del Cauca, Popayán, Colombia ccollazo@unicauca.edu.co

${ }^{3,4}$ Departamento de Informática, Universidad de Lleida, Lleida, España

tonig@diei.udl.cat

rgilediei.udl.cat

\section{ResUMEN}

En el presente artículo se da a conocer una propuesta para la identificación y análisis de las características del comportamiento de los usuarios mientras participan en un proceso de evaluación de sitios web. Las características del comportamiento con la expresión facial, dirección de la mirada, postura, movimientos de la cabeza y gestos. Se presenta un caso de estudio relacionado con la evaluación del sitio web Booking.com, precisando sobre las características del comportamiento identificadas en los usuarios participantes.

Palabras clave: interacción humano - computador, reconocimiento de emociones, factores humanos.

\section{ABSTRACT}

The present article provides a proposal for the identification and analysis of the characteristics of user behavior while participating in a process of evaluation websites. Behavioral characteristics with facial expression, gaze direction, posture, head movements and gestures. A case study related to the evaluation of the Booking.com website, specifying the characteristics of the behavior identified participating users is presented.

Keywords: emotion recognition, human computer interaction, human factors. 


\section{INTRODUCCIÓN}

No es ajeno para nadie que las emociones, inherentes al ser humano, participan de manera importante en las diferentes relaciones de los usuarios con los computadores [1] [2]. Es así que para reconocer las emociones de estos en procesos de evaluación de sistemas interactivos, se aplican cuestionarios que permitan obtener información con respecto a cómo se sienten antes y después de su participación. El uso de dichos cuestionarios se complementa con la observación de los evaluadores o con métodos invasivos. Los resultados de la evaluación se pueden alterar, ya sea por el uso de estos instrumentos o por condicionamientos relacionados con el contexto [3].

Los evaluadores pueden identificar los problemas descritos por los usuarios, pero estos no presentan una interpretación completa si no se entiende lo que el usuario experimenta, siente o espera; la satisfacción hacia un producto no se puede evaluar completamente si no se logran entender los sentimientos internos del usuario [4].

Debido a que las emociones son vistas como "entidades multicomponente", que constan de reacciones expresivas (como por ejemplo sonreír), reacciones fisiológicas (por ejemplo la frecuencia cardiaca) y reacciones del comportamiento (por ejemplo de acercamiento) [5], la información sobre dichas emociones, se puede obtener a través de diferentes fuentes de información: subjetivas, fisiológicas y de comportamiento [6]. Las subjetivas tienen que ver con la información que se obtenga por parte del usuario con respecto a su propio estado emocional, mediante el uso de cuestionarios. Las fisiológicas están relacionadas con identificar las emociones a partir de diferentes cambios fisiológicos y de comportamiento, que para este trabajo específico se tomará considerando características correspondientes a la expresión facial, dirección de la mirada, postura, movimientos de la cabeza y gestos en relación con cambios faciales, corporales y de la voz. Este último será objeto de estudio en la presente investigación.
A partir de las características del comportamiento, se proponen actividades para identificarlas y analizarlas con el propósito de valorar el estado emocional de los usuarios mientras participan en una evaluación en sistemas interactivos.

La sección II presenta conceptos teóricos básicos relacionados con la temática del artículo. En la sección III se presenta información de las actividades propuestas para la identificación e interpretación de las características del comportamiento. En la sección IV se da a conocer un caso de estudio de observación del comportamiento de algunos usuarios evaluando el sitio web Booking. com. Y, finalmente, en la sección $V$ se presentan conclusiones y sugerencias para trabajos futuros.

\section{Base Conceptual}

\section{A. Evaluación emocional}

La evaluación emocional "intenta recopilar y medir información sobre aspectos cualitativos y cuantitativos de la experiencia de un usuario" [7]. Las emociones ayudan a evaluar los resultados y las experiencias de interactuar con productos. Cuando el resultado de la evaluación es positivo surge un sentido de logro y el esfuerzo se reduce o se crea un nuevo objetivo [8].

Los instrumentos de evaluación emocional se pueden clasificar en instrumentos que miden sentimientos subjetivos, reacciones fisiológicas y expresiones emocionales [5],[9].

1) Instrumentos que miden sentimientos subjetivos. Se utilizan autorreportes para que las personas den a conocer sus emociones. Se clasifican en instrumentos de medición verbal, mediante los cuales los encuestados utilizan una escala para el registro de sus emociones, y de medición no verbal, que incluyen representaciones gráficas de la emoción [10]. 
2) Instrumentos de medición fisiológica. Estos instrumentos miden los cambios fisiológicos relacionados con la emoción a través de técnicas no verbales. Los cambios se producen por el ritmo cardiaco, la respiración, la transpiración y la dilatación en la pupila, entre otros [11].

\section{3) Instrumentos que miden expresión} emocional. Se dividen en dos categorías: los que miden la expresión facial y los que miden las expresiones vocales [5]. Los instrumentos que se utilizan para medir la expresión facial están basados en teorías que relacionan las características de la expresión [12]. Una de estas teorías, es el sistema para la expresión facial desarrollado por [13],[14].

Con el propósito de medir los movimientos faciales musculares se creó el Sistema de Codificación de la Acción Facial (FACS, por sus siglas en inglés Facial Action Coding System). FACS se basa en la anatomía humana del rostro y en códigos de expresión en términos de los movimientos, estos últimos llamados "Unidades de Acción" (AUs, por sus siglas en inglés Actions Unity). [13],[14] establecieron 46 AUs, con las cuales describieron cada movimiento de manera independiente. Otra teoría por destacar es la del Sistema de codificación discriminativo al máximo de los movimientos faciales (MAX, por sus siglas en inglés Maximally Discriminative Facial Moving Coding System) [15].

\section{Actividades para LA IDENTIFICACIÓN DE LAS Características Relacionadas CON LA EXPRESIÓN EmOCIONAL}

Se presentan a continuación las actividades propuestas para la identificación e interpretación de la experiencia emocional del usuario, considerando las características relacionadas con su expresión facial, dirección de la mirada, postura, movimientos de la cabeza y gestos, como apoyo a la evaluación emocional de usuarios mientras interactúan con un sitio web.

\section{A. Actividad 1. Establecer las características por identificar.}

En esta actividad se seleccionan las características relacionadas con el comportamiento, que se considere son las más adecuadas para tener en cuenta durante la participación de los usuarios en una evaluación de sistemas interactivos.

Las características seleccionadas corresponden con el Sistema de Codificación del Comportamiento (BCS, por sus siglas en inglés Behavioral Coding System) definido por [16]. BCS [16] propone un extenso listado relacionado con el comportamiento expresivo no verbal de los sujetos. Este sistema está conformado por cuatro diferentes macrocategorías: expresión facial, dirección de la mirada, postura y movimiento de la cabeza y comportamiento vocal. A cada macrocategoría le corresponden ciertas micro categorías que agrupan características propias.

1) Macrocategoría expresión facial. Se relaciona con los diferentes movimientos que se hacen en el rostro. Esta, a su vez, se divide en micro categorías: cara superior, cara inferior y movimiento de los labios. Para cada una de estas micro categorías, el BCS propone utilizar algunas de las unidades de acción planteadas por Ekman y Friesen [14].

Microcategoría cara superior. En la Tabla I se presentan algunas de las características relacionadas con la parte superior de la cara. 
TABLA I

Características Relacionadas con la Cara en la Parte Superior

\begin{tabular}{l|l|l}
$\begin{array}{l}\text { Nomen- } \\
\text { clatura }\end{array}$ & Característica & $\begin{array}{c}\text { Representación } \\
\text { gráfica }\end{array}$ \\
EF-CS-LCl & $\begin{array}{l}\text { El usuario } \\
\text { levanta la ceja } \\
\text { interior. }\end{array}$ \\
\hline $\begin{array}{l}\text { EF-CS- } \\
\text { LCE }\end{array}$ & $\begin{array}{l}\text { El usuario } \\
\text { levanta la ceja } \\
\text { exterior. }\end{array}$ \\
\hline EF-CS-BC & $\begin{array}{l}\text { El usuario baja } \\
\text { las cejas. }\end{array}$ \\
\hline
\end{tabular}

Tomado de [6]

Microcategoría cara inferior. En la Tabla II, se presentan algunas de las características relacionadas con la parte inferior de la cara.

TABLA II

Características Relacionadas con la Cara en la Parte INFERIOR (Tomado de [6])

\begin{tabular}{c|l|c|}
$\begin{array}{c}\text { Nomen- } \\
\text { clatura }\end{array}$ & Característica \\
EF-CI-AN & $\begin{array}{l}\text { El usuario } \\
\text { arruga la nariz. }\end{array}$ \\
EF-CI-LLS & $\begin{array}{l}\text { El usuario realiza } \\
\text { un levantamiento } \\
\text { del labio superior. }\end{array}$ \\
\hline EF-CI-ECL & $\begin{array}{l}\text { El usuario eleva } \\
\text { las comisuras } \\
\text { del labio. }\end{array}$ \\
\hline
\end{tabular}

Microcategoría movimiento de los labios. En la Tabla III se presentan algunas de las características relacionadas con el movimiento de los labios.
TABLA III

Características Relacionadas con el MOVIMIENTO DE LOS LABIOS

\begin{tabular}{|l|l|l|}
$\begin{array}{c}\text { Nomen- } \\
\text { clatura }\end{array}$ & Característica & \multicolumn{1}{c}{$\begin{array}{c}\text { Representación } \\
\text { gráfica }\end{array}$} \\
EF-ML-CL & $\begin{array}{l}\text { El usuario realiza } \\
\text { una contracción } \\
\text { de los labios. } \\
\text { EF-ML-AL }\end{array}$ & $\begin{array}{l}\text { El usuario realiza } \\
\text { un estiramiento } \\
\text { horizontal de } \\
\text { labios. } \\
\text { EHL asuario reación de } \\
\text { los labios. }\end{array}$ \\
\hline
\end{tabular}

Tomado de [6]

2) Macrocategoría dirección de la mirada. Reconocimiento del nivel de atención del usuario, buscando identificar si la atención la dirige o no a la tarea [14]. En la tabla IV se presentan algunas de estas características.

TABLA IV

Características Relacionadas con la DiRECCIÓN DE LA MIRADA

\begin{tabular}{c|l|c|}
$\begin{array}{c}\text { Nomen- } \\
\text { clatura }\end{array}$ & Característica & $\begin{array}{c}\text { Representación } \\
\text { gráfica }\end{array}$ \\
DM-A & $\begin{array}{l}\text { El usuario dirige } \\
\text { la mirada hacia } \\
\text { arriba. }\end{array}$ \\
DM-P & $\begin{array}{l}\text { El usuario dirige } \\
\text { la mirada hacia } \\
\text { el piso. }\end{array}$ \\
DM-R & $\begin{array}{l}\text { El usuario hace } \\
\text { la mirada de } \\
\text { reojo. }\end{array}$ \\
\hline
\end{tabular}

Tomado de [6] 
3) Macrocategoría postura, movimientos de la cabeza y gesto. Se relaciona con los movimientos del cuerpo, de la cabeza y de la cara.

Microcategoría postura. Los movimientos de cuerpo que hace el usuario a partir de la posición inicial; es decir, sentado con la columna derecha en frente del equipo en el cual está dispuesta la evaluación. En la Tabla $V$ se presentan algunas de estas características.

TABLA V

Características Relacionadas con la Postura

\begin{tabular}{c|l}
\multicolumn{1}{c|}{ Nomenclatura } & \multicolumn{1}{c|}{ Característica } \\
PCG-P-PI & $\begin{array}{l}\text { El usuario se encuentra en la posi- } \\
\text { ción inicial; es decir, como se ubicó } \\
\text { al principio de la prueba. Esta es la } \\
\text { posición indicada por el evaluador, } \\
\text { en la cual generalmente se solicita } \\
\text { sentarse en la silla de forma recta. }\end{array}$ \\
PCG-P-AP & $\begin{array}{l}\text { El usuario se acerca a la pantalla, } \\
\text { moviendo el torso y los hombros, } \\
\text { no solamente la cabeza. Se incluyen } \\
\text { los movimientos que empiezan en } \\
\text { la posición inicial y no aquellos que } \\
\text { estando el cuerpo hacia atrás se } \\
\text { mueven hacia adelante. }\end{array}$
\end{tabular}

Tomado de [6]

Micro categoría movimiento de la cabeza. Los movimientos de cabeza que hace el usuario a partir de la posición inicial, generalmente, la cabeza erguida. En la Tabla VI se presentan algunas de estas características.

TABLA VI

Características Relacionadas con el Movimiento de la Cabeza

\begin{tabular}{|l|l|}
\multicolumn{1}{c|}{ Nomenclatura } & \multicolumn{1}{c|}{ Característica } \\
PCG-C-PI & $\begin{array}{l}\text { El usuario sostiene su cabeza en la } \\
\text { posición inicial de la prueba, que ge- } \\
\text { neralmente es mirando hacia el frente } \\
\text { (0 hacia donde le indique el evaluador). } \\
\text { Generalmente, en la posición inicial el } \\
\text { usuario mantendrá la cabeza erguida. } \\
\text { PCG-C-HP }\end{array}$ \\
$\begin{array}{l}\text { El usuario mueve su cabeza hacia } \\
\text { la pantalla. Un cambio se presen- } \\
\text { ta cuando mueve su cabeza con } \\
\text { respecto a la posición inicial. Esta } \\
\text { posición incluye los movimientos que } \\
\text { empiezan en la posición inicial y no } \\
\text { aquellos que estando la cabeza hacia } \\
\text { atrás vuelven a la posición inicial. }\end{array}$ \\
\hline
\end{tabular}


Microcategoría gestos. Son los movimientos que hacen los usuarios con sus manos. En la Tabla VII se presentan algunas de estas características.

TABLA VII

Características Relacionadas con los Gestos

\begin{tabular}{c|l|c|}
$\begin{array}{c}\text { Nomenclatura } \\
\text { PCG-G-TRD }\end{array}$ & $\begin{array}{l}\text { Característica } \\
\text { El usuario toca su rostro } \\
\text { con uno óa dedos. }\end{array}$ \\
PCG-G-CBT & $\begin{array}{l}\text { El usuario cubre su boca } \\
\text { como si fuera a toser. }\end{array}$ \\
\hline
\end{tabular}

Tomado de [6]

A partir de las diferentes características propuestas por el BCS, es necesario seleccionar las que serán objeto de estudio durante la evaluación. Se propone que esta selección se soporte en los heurísticos emocionales [17]; es decir, las pautas para medir con facilidad el estado afectivo sin alto coste [18].
En la Tabla VIII se presenta la relación de algunos de los heurísticos emocionales con las características del BCS. Es importante mencionar que esta relación solo se puede tomar como un referente para identificar el estado emocional del usuario en el momento en que la característica se presenta.

TABLA VIII

Relación de Heurísticos Emocionales con Características del bCS

\begin{tabular}{|c|c|c|}
\hline \multirow{2}{*}{ Heurístico emocional } & \multicolumn{2}{|c|}{ Características del BCS } \\
\hline & Nomenclatura & Representación \\
\hline $\begin{array}{l}\text { Fruncir el ceño. Puede ser } \\
\text { un signo de necesidad de } \\
\text { concentración, desagrado o } \\
\text { percepción de falta de claridad. }\end{array}$ & $\begin{array}{l}\text { EF-CS-BC. El usuario baja } \\
\text { las cejas } \\
\text { EF-Cl-AN. El usuario arruga } \\
\text { la nariz. }\end{array}$ & \\
\hline $\begin{array}{l}\text { Elevar las cejas. Debe } \\
\text { considerarse también una } \\
\text { reacción expresiva negativa. } \\
\text { Elevar las cejas es también } \\
\text { un signo de inseguridad, } \\
\text { incredulidad, sorpresa y } \\
\text { exasperación. }\end{array}$ & $\begin{array}{l}\text { EF-CS-CE. El usuario levanta } \\
\text { la ceja exterior. } \\
\text { EF-CS-SPS. El usuario sube } \\
\text { el parpado superior. }\end{array}$ & \\
\hline
\end{tabular}


Macrocategoría comportamiento vocal. En la Tabla IX se presentan algunas de las características relacionadas con esta macrocategoría.

TABLA IX

Características Relacionadas con el Comportamiento Vocal

\begin{tabular}{|c|c|c|}
\hline Nomenclatura & Nombre & Característica \\
\hline CV-H & Habla & $\begin{array}{l}\text { Los usuarios utilizan lenguaje verbal (oraciones o una sola } \\
\text { palabra). }\end{array}$ \\
\hline CV-NL & No lingüístico & $\begin{array}{l}\text { Se utiliza cuando los usuarios no emiten palabras con } \\
\text { significado, como por ejemplo, exclamaciones. }\end{array}$ \\
\hline CV-R & Risas & Cuando los usuarios se ríen. \\
\hline CV-L & Labial & $\begin{array}{l}\text { Si el usuario mueve sus labios como si estuviera hablando, } \\
\text { pero en realidad no emite sonido alguno. }\end{array}$ \\
\hline CV-O & Otro & Otros sonidos como bostezar, llorar, jadear, etc. \\
\hline
\end{tabular}

Tomado de [6]

Una vez seleccionadas las características, evaluación. En la tabla $X$ se presenta el formato se genera un instrumento para registrar la in- propuesto para la recolección de la información formación sobre las características identifica- (solo se presentará para unas pocas caractedas en los usuarios mientras participan en una rísticas).

TABLA X

Instrumento para la Recolección de Información Sobre las Características del Comportamiento

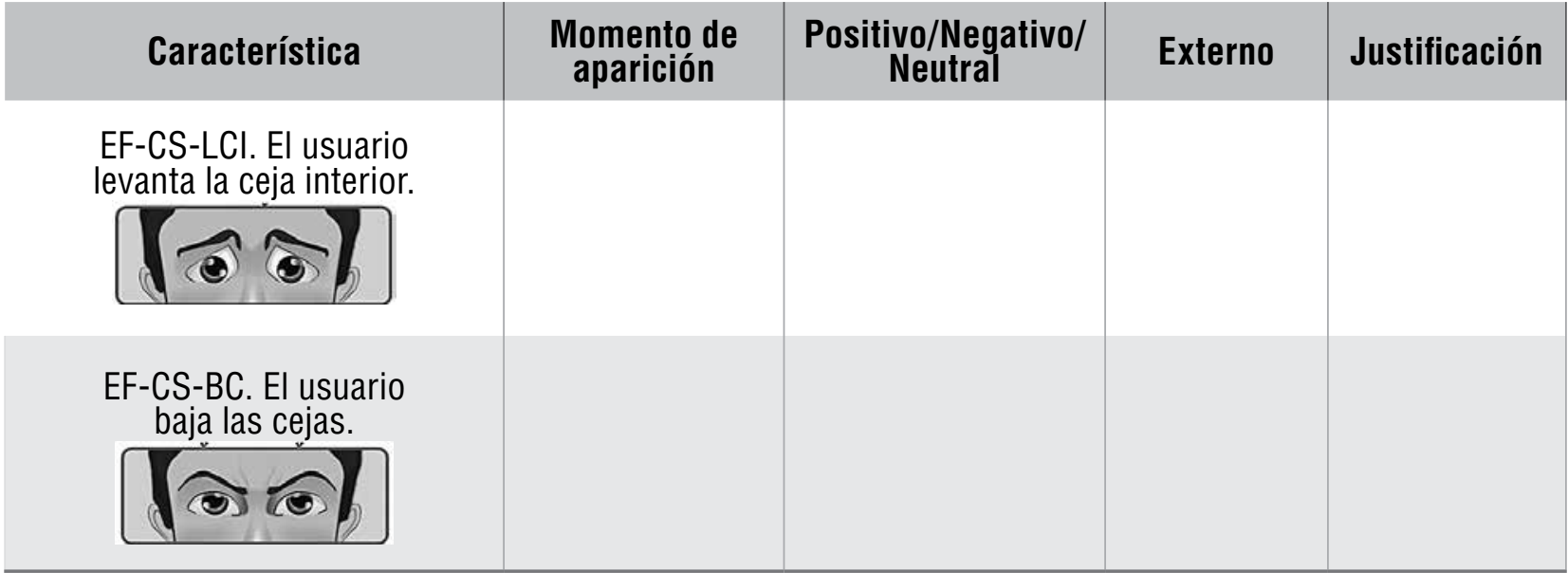

Tomado de [6]

\section{B. Actividad 2. Establecer métricas}

En esta actividad se propone establecer las métricas a partir de las cuales se debe realizar el posterior análisis cuantitativo de la información. Ya que las características del comportamiento son valoradas como positivas, negativas $\mathbf{y}$ neutras, se sugiere hacer uso de la métrica para relacionar el comportamiento verbal con la proporción de comentarios positivos y negativos [19], que para este caso será la proporción de características positivas y negativas. 


\section{Actividad 3. Establecer la tecnología}

En esta actividad se selecciona la tecnología más adecuada para soportar la captura de la información, relacionada con las características del comportamiento.

A partir de las diferentes características del comportamiento planteadas en el BCS, se evidencia que estas se soportan en aspectos faciales, corporales y de expresión vocal, razón por la cual se hace necesario grabar el audio y video del usuario durante su participación en la evaluación, para posteriormente realizar el respectivo análisis.

Son diferentes las tecnologías que se podrían utilizar para este propósito: desde una cámara de video y una aplicación que permita grabar el audio, hasta tecnología como el Kinect [20], mediante el cual es posible detectar movimiento y proximidad, así como el registro de la voz de los usuarios. Así mismo, son variadas las herramientas para la grabación de video y audio mientras se realizan actividades en la pantalla donde se encuentra instalada la cámara web (equipo donde el usuario está realizando la evaluación); la selección de dichas herramientas dependerá de los recursos económicos con los que se disponga y de las características que se requieran. Algunas de las aplicaciones software para la grabación del audio y del video son Camtasia [21], y CamStudio [22], (herramienta de código abierto).

Una alternativa adecuada como apoyo a la tecnología propuesta es el uso de Morae [23], software creado para utilizar en el análisis de estudios de usabilidad y de mercadeo. Este software, además de lo descrito anteriormente, tiene la capacidad de crear, grabar, observar y analizar para grupos focales, investigación de campo y pruebas de productos. Uno de los componentes de Morae es el Morae observer, con el cual es posible incluir anotaciones o marcas (markers[24]) que per- miten, como su nombre lo indica, marcar y anotar información en un punto específico del tiempo de la grabación. Estas anotaciones las puede realizar el observador en el momento exacto de la prueba o posteriormente.

\section{Actividad 4. Adecuar el escenario}

En esta actividad se adecua el escenario donde se realizará la evaluación y se instalan y configuran las herramientas y el software que se requiere para la captura de la información.

\section{E. Actividad 5. Observar el comportamiento del usuario}

Se le pide al usuario que realice la tarea a partir de la cual se requiere identificar su comportamiento. El observador identificará y completará la información en el instrumento de valoración (ver Tabla X), específicamente en los criterios de valoración ausente y presente y en el aspecto momento de aparición.

\section{F. Actividad 6. Valorar las características del comportamiento.}

A partir del entregable de la actividad anterior, se hace necesario completar los demás campos del instrumento de valoración (positivo, negativo, externo) y adicionalmente y muy importante justificar por qué se considera que la característica respectiva es positiva, negativa, neutral o externa. La información que se incluya en el instrumento de valoración es primordial para dar mayor veracidad a los resultados. En la Tabla XI se presenta una propuesta de relación entre algunas de las características del BCS y los criterios de valoración, específicamente los criterios positivo y negativo. Es de anotar que la información de esta tabla solo puede ser tomada como un referente ya que las características presentadas podrían haberse generado por situaciones diferentes a las que se referencian en la columna justificación, soportada en el heurístico emocional. 
TABLA XI

Propuesta de Relación de Características de Comportamiento

y Escalas de Valoración Soportada en los Heurísticos Emocionales

\begin{tabular}{l|l|l}
\multicolumn{1}{c|}{$\begin{array}{c}\text { Nomenclatura } \\
\begin{array}{l}\text { EF-CS-BC. El usuario } \\
\text { baja las cejas }\end{array}\end{array}$} & $\begin{array}{c}\text { Criterio de valoración } \\
\text { (positivo/negativo) }\end{array}$ & \multicolumn{1}{c}{$\begin{array}{c}\text { Justificación soportada } \\
\text { en el heurístico emocional [17] }\end{array}$} \\
$\begin{array}{l}\text { EF-CI-AN. El usuario } \\
\text { arruga la nariz. }\end{array}$ & Negativo & $\begin{array}{l}\text { Heurístico emocional: Fruncir el ceño } \\
\text { El usuario frunció el ceño porque tiene la necesidad } \\
\text { de concentración. } \\
\text { El usuario frunció el ceño porque presentó desagrado. } \\
\text { Se percibe en el usuario alguna falta de claridad. }\end{array}$ \\
\hline $\begin{array}{l}\text { EF-CS-CE. El usuario } \\
\text { levanta la ceja exterior. }\end{array}$ & Negativo & $\begin{array}{l}\text { Heurístico emocional: Elevar las cejas } \\
\text { El usuario eleva las cejas reflejando inseguridad. } \\
\text { El usuario eleva las cejas reflejando incredulidad. } \\
\text { El usuario eleva las cejas en respuesta a una sorpre- } \\
\text { EF-CS-SPS. El usuario } \\
\text { sube el parpado superior. } \\
\text { El usuario eleva las cejas porque se ha exasperado. }\end{array}$ \\
\hline
\end{tabular}

Al respecto de la macrocategoría comportamiento vocal, específicamente para la característica habla (los usuarios utilizan lenguaje verbal (oraciones o una sola palabra)), se sugiere utilizar los resultados de la investigación realizada por Cra-

ggs y Wood [25] en la cual se relacionan algunas etiquetas (nombres de emociones) con las expresiones de los usuarios (ver Tabla XII).

TABLA XII

Relación de Criterio de Valoración (Positivo/Negativo) con Expresiones Vocales de Emociones

\begin{tabular}{|c|c|c|}
\hline \multirow{2}{*}{$\begin{array}{l}\text { Criterio } \\
\text { de valoración }\end{array}$} & \multicolumn{2}{|c|}{ Relación de expresiones vocales con emociones [21] } \\
\hline & Etiqueta de emoción & \begin{tabular}{|l|} 
Expresión \\
\end{tabular} \\
\hline Negativo & Ira/cólera & $\begin{array}{l}\text { El usuario expresa que una situación le ha producido } \\
\text { algún trastorno. }\end{array}$ \\
\hline Externo & Ira/cólera & $\begin{array}{l}\text { El usuario expresa que una persona le ha producido } \\
\text { algún trastorno. }\end{array}$ \\
\hline Positivo & Esperanza (hope) & $\begin{array}{l}\text { El usuario se expresa ya que se anticipa a que alguna } \\
\text { cosa buena está por suceder. }\end{array}$ \\
\hline Positivo & Felicidad & El usuario expresa un sentimiento positivo. \\
\hline Positivo & Positividad & $\begin{array}{l}\text { El usuario expresa el deseo por evitar la tristeza } \\
\text { causada por una situación. Incluye la valentía, deseo } \\
\text { y determinación. }\end{array}$ \\
\hline Externo & Tristeza & $\begin{array}{l}\text { El usuario expresa que una situación, persona o } \\
\text { recuerdo le hace infeliz sin que necesariamente se } \\
\text { sienta motivado para hacer algo al respecto. }\end{array}$ \\
\hline Negativo & Sorpresa desagradable & El usuario expresa que algo inesperado lo ha afectado. \\
\hline Negativo & Preocupación & $\begin{array}{l}\text { El usuario expresa que algo incierto sobre el futuro lo } \\
\text { está afectando negativamente. }\end{array}$ \\
\hline Positivo & Contento & El usuario expresa satisfacción o gratificación. \\
\hline Negativo & Disgusto & $\begin{array}{l}\text { El usuario expresa desaprobación o aversión con } \\
\text { respecto a alguna situación, persona u objeto sin que } \\
\text { se sienta motivado para hacer alguna cosa. }\end{array}$ \\
\hline Externo & Disgusto & $\begin{array}{l}\text { El usuario expresa desaprobación o aversión con res- } \\
\text { pecto a alguna persona sin que se sienta motivado para } \\
\text { hacer alguna cosa. }\end{array}$ \\
\hline
\end{tabular}




\begin{tabular}{|c|c|c|}
\hline \multirow{2}{*}{$\begin{array}{l}\text { Criterio } \\
\text { de valoración }\end{array}$} & \multicolumn{2}{|c|}{ Relación de expresiones vocales con emociones [21] } \\
\hline & Etiqueta de emoción & Expresión \\
\hline Negativo & Frustración & $\begin{array}{l}\text { El usuario expresa su inhabilidad para alcanzar alguna } \\
\text { cosa que lo está afectando. }\end{array}$ \\
\hline Negativo & Miedo & $\begin{array}{l}\text { El usuario expresa una disposición causada por la anti- } \\
\text { cipación de que algo malo le ocurra. }\end{array}$ \\
\hline Positivo & Alegría, placer & $\begin{array}{l}\text { El usuario expresa un sentimiento positivo con el cual } \\
\text { intenta disfrutar. }\end{array}$ \\
\hline Negativo & $\begin{array}{l}\text { Miseria, sufrimiento, } \\
\text { desdicha }\end{array}$ & $\begin{array}{l}\text { El usuario expresa una situación que lo está haciendo } \\
\text { infeliz sin que necesariamente se sienta motivado a } \\
\text { hacer algo sobre esto. }\end{array}$ \\
\hline
\end{tabular}

Tomado de [6]

\section{G. Actividad 7. Análisis de los datos de ma- nera cuantitativa}

Una vez se tiene información completa en el instrumento de valoración (ver Tabla $\mathrm{X}$ ) sobre los diferentes usuarios que hayan participado en la evaluación, se realiza el análisis cuantitativo de los datos.
El instrumento de valoración tiene información correspondiente a las características identificadas del comportamiento. Se propone utilizar un formato como el presentado en la Tabla XIII, el cual debe completarse por cada una de las tareas realizadas por los usuarios y para cada uno de ellos.

TABLA XIII

Formato para Realizar Conteo de las Características del Comportamiento por Tarea Según Criterio de Valoración (Positivo, Negativo, Neutro)

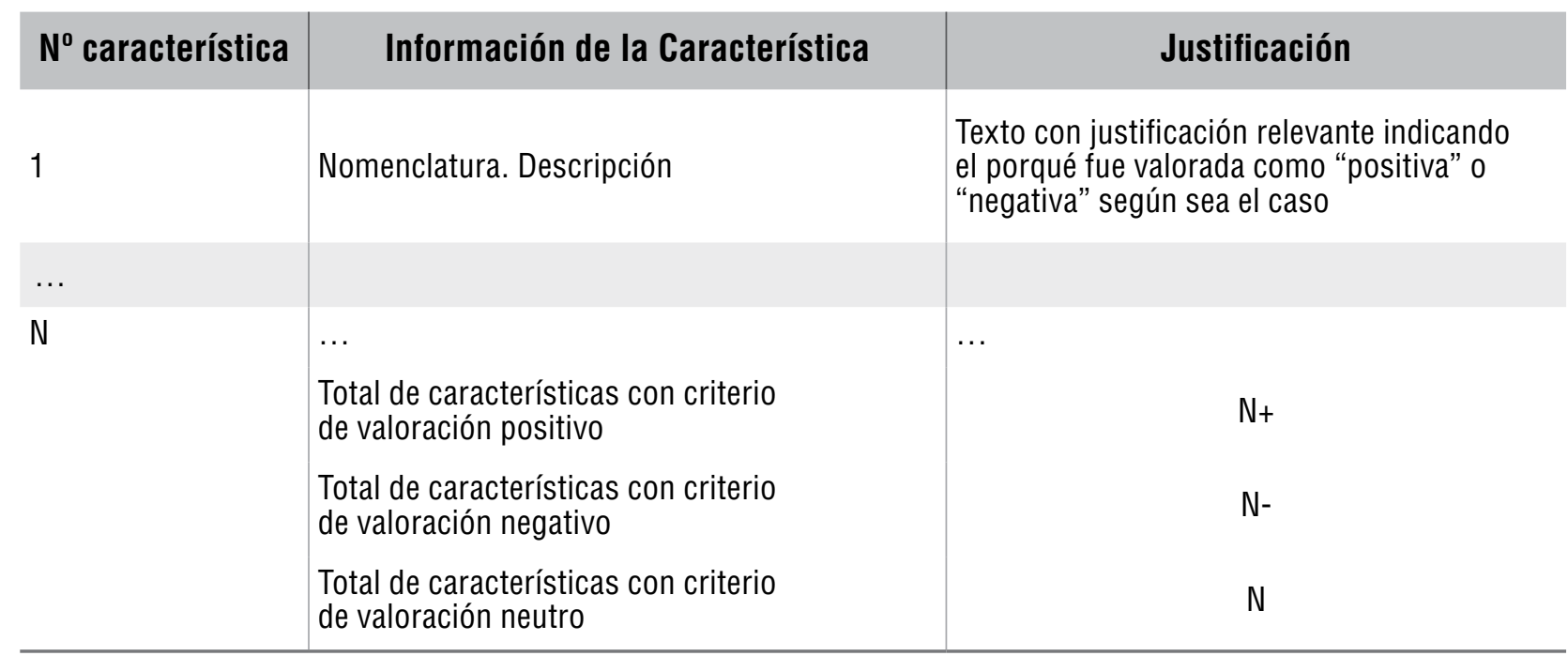

Tomado de [6]

A partir de la información recolectada en la Tabla XIII, se conoce la cantidad de características positivas y negativas identificadas, como se presenta en la Tabla XIV. 
TABLA XIV

FORMATO DE RESUMEN, CARACTERÍSTICAS POSITIVAS Y NEGATIVAS

\begin{tabular}{l|c}
\multicolumn{1}{c|}{ Características } & Frecuencia relativa \\
Positivas & \\
Característica 1 & Valor \\
$\ldots$ & $\ldots$ \\
\hline Negativas & \\
Característica 1 & Valor \\
$\ldots$ & $\ldots$ \\
\hline
\end{tabular}

Tomado de [6]

Es importante mencionar que el determinar que una tarea tuvo más características positivas que negativas no dice mucho por sí misma; tendrá mucho más significado si las proporciones son comparadas entre diferentes diseños o productos [19].

\section{H. Actividad 8. Análisis de los datos de manera cualitativa}

A partir de la información recolectada en la actividad análisis cuantitativo de los datos y la relación que se haya establecido con los heurísticos emocionales y con las expresiones vocales, se realiza el análisis cualitativo. La información que se ha de presentar se debe dar en términos de las posibles relaciones entre las características y los heurísticos emocionales.

\section{Caso de Estudio. Valoración de Características del Comportamiento en la Evaluación del Sitio Web BOOKING.COM}

\section{A. Objetivo}

Obtener información sobre el estado emocional de 6 estudiantes universitarios con rango de edad entre los 22 y 32 años (con un promedio de 26 años), a partir de las características de comportamiento de cada uno de ellos, durante una evaluación de sistemas interactivos, específicamente del sitio web booking.com.

\section{B. Procedimiento}

La evaluación del sitio web Booking.com se realizó mediante el método de evaluación de usabilidad, el conductor, que consiste en que un evaluador guía al usuario en la realización de las actividades, dando respuesta a las preguntas del participante relacionadas con el uso del sistema [26]. Las tareas realizadas por los usuarios fueron tres: T1. Modificar una reserva; T2. Cancelar la reserva y T3. Explorar la opción consultas recientes. Antes de realizar las tareas en el sitio web y con el propósito de tranquilizar a los usuarios, se les presentó un video con imágenes de la naturaleza y sonidos relajantes.

El evaluador observó y completó la información en el instrumento de valoración, específicamente en los criterios de valoración ausente y presente y el aspecto momento de aparición, a partir de las características del comportamiento identificadas en los usuarios. Esto se hizo para cada una de las tareas que cada uno realizó. Seguidamente se dio una valoración a cada una de las características del comportamiento.

Una vez se tiene información completa en el instrumento de valoración de los diferentes usuarios que participan en la evaluación, se presentan los datos de manera cuantitativa, con base en la métrica establecida (proporción de características positivas y negativas).

\section{Análisis de datos}

Se presenta a continuación el análisis de datos para la tarea modificar información de la reserva. Al respecto, se conoce con anticipación que se relaciona con un estado emocional negativo por parte de los usuarios ya que no se encuentra en el sitio web Booking.com opción para modificar. La única manera para que los usuarios modifiquen la información es utilizar la opción de regresar (flecha con dirección a la izquierda ubicada en la parte superior izquierda) que se presenta en el navegador ( Fig 1.) 
Fig. 1 Indicación de opción disponible en el sitio web para modificar información de la reserva.

En la Tabla XV se presenta información de algunas de las características identificadas en el com- portamiento de los usuarios mientras participaron en la tarea de modificar información de la reserva.

TABLA XV

Conteo de las Características del Comportamiento.

Momento: Realizando Tarea de Modificar Información de la Reserva

\begin{tabular}{|c|c|c|c|c|}
\hline Usuario & Característica & $\begin{array}{l}\text { Momento } \\
\text { de aparición }\end{array}$ & Justificación & Valoración \\
\hline 1 & $\begin{array}{l}\text { W (Gestos) .PCG-G-3: el } \\
\text { usuario cubre su boca } \\
\text { como si fuera a toser. }\end{array}$ & $\begin{array}{l}\text { Buscando la opción } \\
\text { para modificar el } \\
\text { nombre. }\end{array}$ & $\begin{array}{l}\text { El usuario no encuentra al- } \\
\text { guna opción para modificar } \\
\text { los datos ingresados. }\end{array}$ & Negativo \\
\hline 1 & $\begin{array}{l}\text { A (Otras no especificadas). } \\
\text { El usuario sube el labio } \\
\text { derecho. }\end{array}$ & Ingresando los datos. & $\begin{array}{l}\text { Está ingresando los datos, } \\
\text { no se evidencia un estado } \\
\text { emocional positivo o } \\
\text { negativo específico }\end{array}$ & Neutro \\
\hline 1 & $\begin{array}{l}\text { W (Gestos) .PCG-G-3. EI } \\
\text { usuario cubre su cara como } \\
\text { si fuera a toser. }\end{array}$ & $\begin{array}{l}\text { Cuando el usuario ter- } \\
\text { mina de incluir la infor- } \\
\text { mación por modificar. }\end{array}$ & $\begin{array}{l}\text { No se evidencia si es un } \\
\text { estado emocional positivo } \\
\text { o negativo. }\end{array}$ & Neutro \\
\hline 2 & $\begin{array}{l}\text { A (otras no especificadas) } \\
\text {.El usuario inclina la cabeza. } \\
\text { Apreciación: negativo }\end{array}$ & $\begin{array}{l}\text { Mientras busca la } \\
\text { opción para modificar }\end{array}$ & $\begin{array}{l}\text { El usuario no encuentra } \\
\text { la opción para modificar. }\end{array}$ & Negativo \\
\hline 2 & $\begin{array}{l}\text { F (Habla). ¿Es para evaluar } \\
\text { este sitio?' }\end{array}$ & $\begin{array}{l}\text { Mientras busca la } \\
\text { opción para modificar. }\end{array}$ & $\begin{array}{l}\text { El usuario no encuentra } \\
\text { la opción para modificar. }\end{array}$ & Neutro \\
\hline 2 & F (Habla).'Yo no lo veo' & $\begin{array}{l}\text { Mientras busca la } \\
\text { opción para modificar. }\end{array}$ & $\begin{array}{l}\text { El usuario no encuentra } \\
\text { la opción para modificar }\end{array}$ & Negativo \\
\hline 2 & $\begin{array}{l}\text { F (Habla).'Es que estoy } \\
\text { ciego?' }\end{array}$ & $\begin{array}{l}\text { Mientras busca la } \\
\text { opción para modificar. }\end{array}$ & $\begin{array}{l}\text { El usuario no encuentra } \\
\text { la opción para modificar. }\end{array}$ & Negativo \\
\hline 2 & F (Habla) .mmmmmmmmm & $\begin{array}{l}\text { Mientras busca la } \\
\text { opción para modificar. }\end{array}$ & $\begin{array}{l}\text { El usuario no encuentra } \\
\text { la opción para modificar. }\end{array}$ & Negativo \\
\hline
\end{tabular}

En la Tabla XVI se presenta un resumen de las características identificadas y mostradas en la Tabla XV. 
TABLA XVI

INFORMACIÓN CUANTITATIVA DE CARACTERÍSTICAS. MOMENTO:

REALIZANDO TAREA DE MODIFICAR INFORMACIÓN DE LA RESERVA.

\begin{tabular}{c|c|c|c|c|c|c|c|}
\cline { 2 - 6 } N` Usuario & \multicolumn{2}{|c|}{$\begin{array}{c}\text { Positivas } \\
\text { Frecuencia }\end{array}$} & \multicolumn{2}{c|}{$\begin{array}{c}\text { Negativas } \\
\text { Frecuencia }\end{array}$} & \multicolumn{2}{c|}{$\begin{array}{c}\text { Neutras } \\
\text { Frecuencia }\end{array}$} & \multirow{2}{*}{ Total } \\
\cline { 2 - 7 } & Absoluta & Porcentual & Absoluta & Porcentual & Absoluta & Porcentual & \\
\hline 1 & 0 & $0,00 \%$ & 1 & $33,33 \%$ & 2 & $66,67 \%$ & 3 \\
\hline 2 & 0 & $0,00 \%$ & 5 & $83,33 \%$ & 1 & $16,67 \%$ & 6 \\
\hline 3 & 0 & $0,00 \%$ & 4 & $80,00 \%$ & 1 & $20,00 \%$ & 5 \\
\hline 4 & 0 & $0,00 \%$ & 5 & $55,56 \%$ & 4 & $44,44 \%$ & 9 \\
\hline 5 & 0 & $0,00 \%$ & 2 & $50,00 \%$ & 2 & $50,00 \%$ & 4 \\
\hline 6 & 0 & $0,00 \%$ & 9 & $81,82 \%$ & 2 & $18,18 \%$ & 11 \\
\hline
\end{tabular}

En la Fig. 2 se presenta el histograma de frecuencia porcentual de las características posi-

usuarios durante la ejecución de la tarea modifitivas, negativas y neutras, identificadas en los

car información de la reserva.

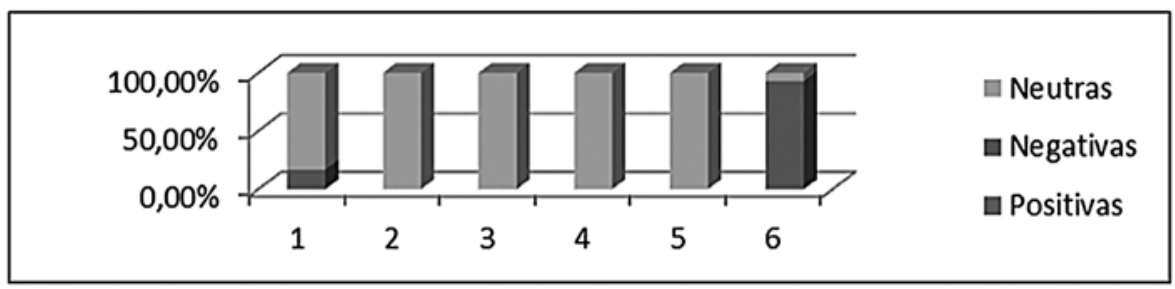

Fig. 2 Representación de las proporciones entre las características para todas las tareas.

Teniendo en cuenta la información anterior, se concluye:

\section{Usuario 1}

No se identificaron características valoradas como positivas.

Se identificaron dos características valoradas como neutras, las cuales no se establecieron ni como positivas ni como negativas ya que el usuario solo estaba completando información en ese justo momento.

La característica valorada como negativa se presentó mientras buscaba la opción para modificar la reserva.

\section{Usuario 2}

No se identificaron características valoradas como positivas.

Se identificó una característica valorada como neutra, que tiene relación con la expresión oral de "¿es para evaluar este sitio?”.

Se identificaron cinco características valoradas como negativas las cuales estuvieron presentes mientras el usuario buscaba la opción para modificar la reserva. 


\section{Usuario 3}

No se identificaron características valoradas como positivas.

Se identificó una característica (eleva las comisuras del labio) valorada como neutra justo al iniciar la tarea.

Las características valoradas como negativas se evidenciaron cuando el usuario estaba buscando la opción para modificar la reserva.

\section{Usuario 4}

No se identificaron características valoradas como positivas.

Se identificaron 3 características valoradas como neutras.

Las características valoradas como negativas se evidenciaron cuando el usuario estaba buscando la opción para modificar la reserva.

\section{Usuario5}

No se identificaron características valoradas como positivas.

Se identificaron 2 características valoradas como neutras. No se valoraron ni como negativas ni como positivas ya que se identificaron justo al iniciar la tarea.
Las características valoradas como negativas se evidenciaron cuando el usuario estaba buscando la opción para modificar la reserva. Ya llevaba un tiempo considerable.

\section{Usuario 6}

No se identificaron características valoradas como positivas.

Se identificaron dos características valoradas como neutras. Una de estas no se valoró ni como negativa ni como positiva ya que se identificaron justo al iniciar la tarea. La otra característica fue por un suceso externo (el usuario llevó la boca a la mano para toser).

Las características valoradas como negativas se evidenciaron cuando el usuario estaba buscando la opción para modificar la reserva. Ya llevaba un tiempo considerable.

En la Tabla XVII se presenta información de la relación entre algunas de las características, la valoración y los heurísticos emocionales. La información de la relación entre las características y los heurísticos es una aproximación al estado emocional del usuario.

TABLA XVII

Relación de Características y Heurísticos Emocionales. Momento:

Realizando Tarea de Modificar Información de la Reserva.

\begin{tabular}{|c|c|c|c|c|}
\hline Característica & Justificación & $\begin{array}{l}\text { Valoración/ } \\
\text { cantidad }\end{array}$ & $\begin{array}{l}\text { Heurístico } \\
\text { emocional }\end{array}$ & Descripción del heurístico \\
\hline \multicolumn{5}{|c|}{ Usuario 1} \\
\hline $\begin{array}{l}\text { 1- W (Gestos) } \\
\text { PCG-G-3: El } \\
\text { usuario cubre } \\
\text { su boca como si } \\
\text { fuera a toser. }\end{array}$ & $\begin{array}{l}\text { El usuario no } \\
\text { encuentra alguna } \\
\text { opción para mo- } \\
\text { dificar los datos } \\
\text { ingresados }\end{array}$ & Negativo/1 & $\begin{array}{l}\text { Tocarse la cara } \\
\text { con las manos. }\end{array}$ & $\begin{array}{l}\text { El usuario eleva la mano que esta- } \\
\text { ba sobre el ratón, como un signo } \\
\text { de confusión e incertidumbre. Se } \\
\text { puede relacionar con que el usua- } \\
\text { rio se siente perdido o cansado. }\end{array}$ \\
\hline \multicolumn{5}{|c|}{ Usuario 2} \\
\hline $\begin{array}{l}\text { A (Otras no } \\
\text { especificadas) .El } \\
\text { usuario inclina la } \\
\text { cabeza. }\end{array}$ & $\begin{array}{l}\text { El usuario no en- } \\
\text { cuentra la opción } \\
\text { para modificar }\end{array}$ & Negativo/1 & $\begin{array}{l}\text { Inclinar el } \\
\text { cuerpo hacia } \\
\text { adelante. }\end{array}$ & $\begin{array}{l}\text { El usuario se inclina hacia adelan- } \\
\text { te como un signo de depresión } \\
\text { y frustración relacionado con el } \\
\text { trabajo que se tiene entre manos. }\end{array}$ \\
\hline
\end{tabular}


En la Tabla XVIII se presenta información de la relación entre las características y los resultados de la investigación realizada por [25], en la cual se encuentran algunas etiquetas (nombres de emociones) con las expresiones realizadas por los usuarios. Esta relación es una aproximación al estado emocional del usuario.

\section{TABLA XVIII}

Relación De Características Etiquetas de Emociones Identificadas A PartiR De Las Expresiones Orales. Momento: Realizando Tarea De Modificar Información De La Reserva.

\begin{tabular}{|c|c|c|c|}
\hline $\begin{array}{c}\text { Característica/ } \\
\text { Justificación }\end{array}$ & $\begin{array}{c}\text { Valoración/ } \\
\text { Cantidad }\end{array}$ & $\begin{array}{l}\text { Etiqueta de } \\
\text { la emoción }\end{array}$ & Expresión \\
\hline \multicolumn{4}{|c|}{ Usuario 2} \\
\hline $\begin{array}{l}\text { F (Habla).'Yo no lo veo'. } \\
\text { El usuario no encuentra la } \\
\text { opción para modificar }\end{array}$ & Negativo/1 & Disgusto & $\begin{array}{l}\text { "El usuario expresa desapro- } \\
\text { bación o aversión con respecto } \\
\text { a alguna situación, persona u } \\
\text { objeto sin que se sienta motivado } \\
\text { para hacer alguna cosa." }\end{array}$ \\
\hline $\begin{array}{l}F \text { (Habla).'Es que estoy } \\
\text { ciego?' } \\
\text { El usuario no encuentra la } \\
\text { opción para modificar. }\end{array}$ & Negativo/1 & Frustración & $\begin{array}{l}\text { "El usuario expresa su inhabilidad } \\
\text { para alcanzar alguna cosa que lo } \\
\text { está afectando" }\end{array}$ \\
\hline $\begin{array}{l}\text { F (Habla) .mmmmmmmm. } \\
\text { El usuario no encuentra la } \\
\text { opción para modificar. } \\
\text { F (Habla). Se ríe porque no } \\
\text { ha encontrado la opción. El } \\
\text { usuario no encuentra la opción } \\
\text { para modificar }\end{array}$ & Negativo/1 & $\begin{array}{c}\text { Sorpresa } \\
\text { desagradable. }\end{array}$ & $\begin{array}{l}\text { Sin establecer. } \\
\text { El usuario expresa que algo } \\
\text { inesperado lo ha afectado. }\end{array}$ \\
\hline \multicolumn{4}{|c|}{ Usuario 3} \\
\hline $\begin{array}{l}\mathrm{F} \text { (Habla) .mmmmmm. Podría. } \\
\text { relacionarse con que no } \\
\text { encuentra la información }\end{array}$ & Negativo/1 & $\begin{array}{c}\text { Sorpresa } \\
\text { desagradable }\end{array}$ & $\begin{array}{l}\text { Sin establecer. } \\
\text { El usuario expresa que algo } \\
\text { inesperado lo ha afectado. }\end{array}$ \\
\hline
\end{tabular}

\section{Conclusiones}

La información presentada sobre el caso de estudio no es contundente con respecto a la propuesta de integración de las actividades para valoración del comportamiento ya que se llevó a cabo con solo seis participantes. Sin embargo, posibilitó la puesta en marcha de la integración de las actividades como alternativa para complementar la evaluación del uso de sistemas interactivos. Se requiere la realización de diferentes casos de estudio para validar de manera más adecuada la integración de las actividades.
El reconocimiento de las características del comportamiento y su posible relación con el estado emocional del usuario ofrece una información inicial para tener conocimiento sobre la experiencia que este tuvo con el sistema mientras realizaba las tareas propias de la evaluación. Los heurísticos emocionales proponen una forma de relacionar las características con el estado emocional de los usuarios. Sin embargo, esta valoración debe ser soportada por el momento de aparición de la característica ya que se sugiere que algunas de estas 
se valoren, con base en los heurísticos, como negativas; sin embargo, pueden presentarse en situaciones en las cuales no necesariamente haya algún evento que propicie un estado emocional negativo en el usuario. De ahí, que la propuesta de valorar las características como negativas o positivas soportado en el heurístico emocional, debe ser tomado solo como un referente que se sustenta con el propio contexto de la evaluación.

Al utilizar únicamente el análisis de las características del comportamiento no se puede llegar a una conclusión general sobre la experiencia emocional presentada por los usuarios mientras interactúan con un sistema interactivo, ya que se evidenciaron casos en los cuales algunos usuarios

\section{REFERENCIAS}

[1] C. Peter, R. Beale, E. Crane, and L. Axelrod, "Emotion in $\mathrm{HCl}$," in Proceedings of the 21st British $\mathrm{HCl}$ Group Annual Conference on People and Computers: HCl... but not as we know it - Volume 2, 2007, pp. 211-212.

[2] S. Brave and C. Nass, "Emotion in Human-Computer Interaction." in The Human-Computer Interaction Handbook. Fundamentals, Evolving Technologies and Emerging Applications., Second., vol. 29, no. 2, A. Sears and J. A. Jacko, Eds. Standford University: Lawrence Erlbaum Associates, 2008, p. 1386.

[3] R. Picard and S. Daily, "Evaluating affective interactions: Alternatives to asking what users feel," $\mathrm{CHI}$ Work. Eval. Affect. ..., 2005.

[4] B. Zaman, "The FaceReader: Measuring instant fun of use," 4th Nord. Conf. Human-, no. October, pp. 457-460, 2006.

[5] P. Desmet, "Designing emotions," Delft University of Technology, Dept. of Industrial Design], 2002.

[6] Y. Mendez-Alegria, "Marco de referencia para la captura semi-invasiva y análisis de las emociones en el proceso de evaluación con usuarios de sistemas interactivos," Universidad del Cauca.

[7] C. González, M. Cairós-González, and Vicente Navarro-Adelantado, "EMODIANA: Un instrumento para la evaluación subjetiva de emociones en niños y niñas," in CEDI2013 IV Congreso Español de Informatica, 2013.

[8] J. Forlizzi and K. Battarbee, "Understanding Experience in Interactive Systems," in Proceedings of the 5th Conference on Designing Interactive Systems: Processes, Practices, Methods, and Techniques, 2004, pp. 261-268. no son muy expresivos y no fue posible identificar características del comportamiento. Tendría un mayor significado, si por ejemplo, para el mismo usuario se presentan diferentes propuestas de diseño.

La valoración (positivo, negativo y neutral) que se le da a cada una de las características del comportamiento, tiene un alto grado de subjetividad ya que depende del criterio del observador, razón por la cual se hace necesario complementar esta información con los resultados obtenidos mediante la evaluación emocional, soportada en el análisis de la actividad cerebral (fisiológico) y en la propia información (subjetivo) que den los usuarios sobre su experiencia emocional.

[9] R. G. Mendez-Alegria, Yenny, Cesar Collazos, Toni Granollers, "Rueda de Emociones de Ginebra+: instrumento para la evaluación emocional de los usuarios mientras participan en una evaluación de sistemas interactivos," Rev. Ing. Dyna, vol. En prepara, 2015.

[10] A. Agarwal and A. Meyer, "Beyond usability: evaluating emotional response as an integral part of the user experience," in $\mathrm{CHI} 09$ Proceedings of the 27th international conference extended abstracts on Human factors in computing systems, 2009, pp. 2919-2930.

[11] R. Hartson and P. Pyla, The UX Book: Process and Guidelines for Ensuring a Quality User Experience, 1st ed. San Francisco, CA, USA: Morgan Kaufmann Publishers Inc., 2012.

[12] P. Desmet, "Measuring emotion: development and application of an instrument to measure emotional responses to products," in Funology, vol. 41, no. 4, M. A. Blythe, K. Overbeeke, A. F. Monk, and P. C. Wright, Eds. Kluwer Academic Publishers, 2004, pp. 111-123.

[13] P. Ekman and W. Friesen, "Measuring facial movement," Environ. Psychol. Nonverbal Behav., pp. 5675, 1976.

[14] P. Ekman and W. Friesen, The Facial Action Coding System. Palo Alto: CA: Consulting Psychological Press, 1978.

[15] C. E. Izard, The Maximally Discriminative Facial Movement Coding System (MAX). Newark, Del: University of Delaware, Instructional Resource Center, 1979.

[16] Ciceri, Colombo, and Balzarotti, "Behavioral Coding System," 2005. 
[17] E. De Lera and M. Garreta-domingo, "Ten Emotion Heuristics : Guidelines for assessing the user's affective dimension easily and cost-effectively," Group, vol. 2, no. September, pp. 7-10, 2007.

[18] E. D. y M. G.-D. Lera, "10 heurísticos emocionales. Pautas para evaluar la dimensión afectiva de los usuarios de forma fácil y económica," Diseño e Interacción Faz, vol. 2, pp. 68-79, 2008.

[19] T. Tullis and B. Albert, "Behavioral and Physiological Metrics," in Measuring the User Experience, Elsevier, 2013, pp. 163-186.

[20] http://www.microsoft.com/en-us/kinectforwindows/ revisado julio del 2013.
[21] http://www.techsmith.com/camtasia.html.

[22] http://camstudio.org/ revisado junio del 2013.

[23] Morae, "Morae," 2014. [Online]. Available: http://www. techsmith.com/morae.html, revisado julio del 2013.

[24] http://www.techsmith.com/tutorial-morae-tasks-markers-in-manager-prior.html, revisado junio del 2013.

[25] R. Craggs and M. Wood, "A categorical annotation scheme for emotion in the linguistic content of dialogue," Affect. dialogue Syst., 2004, revisado junio del 2013.

[26] J. Nielsen, Usability Engineering. Academic Press, 1993, revisado junio del 2013. 
